\title{
Diagnostic use of computerized axiography in TMJ disc displacements
}

\author{
DANIEL TALMACEANU ${ }^{1}$, NICOLAE BOLOG ${ }^{2,3}$, DANIEL LEUCUTA ${ }^{4}$, \\ IOAN ANDREI TIG ${ }^{5}$ and SMARANDA BUDURU ${ }^{6}$ \\ ${ }^{1}$ Department of Oral Surgery, 'Stomestet' Dental Clinic, 400372 Cluj-Napoca, Romania; \\ ${ }^{2}$ Department of Radiology, Phoenix Swiss Med GmbH, 4153 Reinach, Switzerland; \\ ${ }^{3}$ Skyra Vision Imaging Center, 400370 Cluj-Napoca; ${ }^{4}$ Department of Medical Informatics and Biostatistics, \\ 'Iuliu Haţieganu' University of Medicine and Pharmacy, 400349 Cluj-Napoca; ${ }^{5}$ Department of Dental Medicine, \\ Faculty of Medicine and Pharmacy, University of Oradea, 410563 Oradea; ${ }^{6}$ Department of Prosthodontics, \\ 'Iuliu Haţieganu' University of Medicine and Pharmacy, 400349 Cluj-Napoca, Romania
}

Received October 6, 2021; Accepted November 5, 2021

DOI: $10.3892 / \mathrm{etm} .2022 .11137$

\begin{abstract}
Application of paraclinical methods for investigating the temporomandibular joint (TMJ) has been a subject of constant controversy due to the absence of universally-accepted criteria and lack of consensus regarding their usage. Compared with medical imaging, which provides a structural analysis of the TMJ, axiography involves the functional recording of condylar movements. The aim of the present study was to explore the diagnostic value of computerized axiography for TMJ disc displacements using MRI as the reference standard. The present study included 33 (66 TMJs) patients clinically diagnosed with TMJ disc displacements. On the same day, all patients underwent clinical examination and computerized axiography measurements using Cadiax Compact ${ }^{\circledR}$ II before undergoing MRI (1.5 T) 1-7 days later. The characteristics of the diagnostic parameters, namely sensitivity, specificity, positive and negative predictive values, accuracy, Youden index and the 95\% confidence intervals (CI), were all computed. Compared with MRI, computerized axiography yielded a sensitivity of $85.11 \%$, specificity of $94.74 \%$, positive predictive value of $97.56 \%$, negative predictive value of $72 \%$ and a diagnostic accuracy of $87.88 \%$ for any disc displacements. This suggests that computerized axiography can confer high sensitivity, specificity and accuracy for the diagnosis of TMJ disc displacements. However, axiographic analysis has
\end{abstract}

Correspondence to: Dr Ioan Andrei Tig, Department of Dental Medicine, Faculty of Medicine and Pharmacy, University of Oradea, Strada Cerbului 1, 410563 Oradea, Romania

E-mail: nelutig@yahoo.com

Key words: temporomandibular joint, temporomandibular disorders, disc displacement, computerized axiography, magnetic resonance imaging no diagnostic significance in TMJ function if not associated with clinical examination.

\section{Introduction}

The potential application of paraclinical methods for investigating the temporomandibular joint (TMJ) has been a subject of constant controversy due to the absence of definitive criteria and the lack of consensus regarding usage. The mechanism underlying mandibular movements is highly complex and it is mandatory that novel examination methods are developed to further the understanding in the function of this joint. Apart from clinical evaluation and medical imaging, graphical recordings of mandibular movements obtained using computerized axiography can make a significant contribution to the functional evaluation of the TMJ (1). Compared with medical imaging, which provides a structural analysis of the TMJ, axiography consists of making functional recordings of condylar movements (2). Initially implemented as a mechanical measurement method, axiography is also called condylography and is currently being applied on digital platforms (3).

Computerized axiography reveals information on the opening, closing, lateral and protrusive movements $(2,3)$, where its graphical output can be analyzed in the horizontal as well as the sagittal planes (4). This method also enables the quantitative and qualitative analysis of pathways in both the orbiting and pivoting condyle in terms of symmetry, speed during articular movements and aspect of the condylar pathway (5). In addition to information on the optimal or suboptimal TMJ performance, computerized axiography provides data required to program the semi-adjustable articulator, which includes Bennett angle values and the sagittal condylar inclination (6). This characteristic of computerized axiography have proven useful in the dental treatment industry, including prosthetic, orthodontic and orthognathic surgery, all of which requires the faithful replication of musculo-articular dynamics data of the dento-maxillary system (7). 
The aim of the present study was to determine the accuracy of using computerized axiography for diagnosing TMJ displacements, using MRI as the reference standard. In addition, another objective of the present study was to calculate the mean values of the Bennett angle and sagittal condylar inclination and determine their variations according to the pathology of each case examined.

\section{Patients and methods}

Patients. In total, 33 (66 TMJs) with signs and symptoms of TMJ disc displacement according to the Research Diagnostic Criteria for Temporomandibular Disorders (RDC/TMD) (8) were enrolled in this prospective study performed over 6 months (between May and October 2017). Of the 33 patients enrolled in the present study, there were five males and 28 females. The age of the patients ranged from 14 to 65 , with a mean \pm SD age of $28.4 \pm 11.3$ years. On the same day, each patient underwent clinical examination and computerized axiography, which was performed by a single clinician (DT). MRI was performed 1-7 days after clinical examination. The clinical examination and computerized axiography were performed at 'Stomestet' Dental Clinic (Cluj-Napoca, Romania), whereas MRI was performed at 'Skyra Vision' Imaging Center (Cluj-Napoca, Romania). Only patients with the following clinical TMJ disorders were included in the study: Disc displacement with reduction (DDwR) and disc displacement without reduction (DDwoR). The following signs were necessary for DDwR diagnosis: i) Joint noise reported by patient; ii) click detection during opening/closing cycles; and iii) click detection during lateral and protrusive movements. For DDwoR diagnosis, the following signs were considered: i) Unassisted opening $<35 \mathrm{~mm}$ and assisted opening $<4 \mathrm{~mm}$ more than the unassisted opening; ii) contralateral movements $<7 \mathrm{~mm}$ and/or uncorrected deviation to the ipsilateral side on opening; and iii) absence of clicking noise (8).

Patients with contraindication to MRI, including claustrophobia, cardiac pacemakers or ferromagnetic metals carriers, those who were unable to undergo computerized axiography due to muscular instability or inability to correctly perform mandibular movements, in addition to patients with masticatory muscles disorders, were excluded.

The research protocol in the present study was analyzed and approved by the Ethics Committee of the University of Medicine and Pharmacy 'Iuliu Hatieganu' Cluj-Napoca (approval no. 403/02.07.2015). Written informed consent was obtained from each participant or guardian/parent of the participant enrolled in the study.

Clinical examination. Clinical examination was performed by a single oral surgeon (DT). Diagnosis was made based on the patient's history and physical examination. The clinician investigated the masticatory and cervical muscles and both TMJs. Dental occlusion was also analyzed, both static and dynamic. The diagnostic decision tree was elaborated according to the RDC/TMD (8).

Computerized axiography. Computerized axiography and clinical examination were performed on the same day by the same specialist. Axiography was performed using the Cadiax
Compact $^{\circledR}$ II device (GAMMA Medizinisch-wissenschaftliche Fortbildungs $\mathrm{GmbH}$ ). Prior to examination, each patient was informed about the movements required during the examination. The Cadiax Compact ${ }^{\circledR}$ II device consists of the following elements: A face bow; a mandibular bow; a bite fork; two stilettos; two recording sensors; a central unit; and a computer with the required software (Figs. 1 and 2; Cadiax Compact 2 Version 2.9.2; GAMMA Medizinisch-wissenschaftliche Fortbildungs GmbHJ) (5).

Recording mandibular movements. The reference position, which is the starting point for all the movements, was always recorded first. This is followed by maximum protrusion measurements, right laterotrusion measurements, left laterotrusion measurements and maximum opening, in that order. The recording is immediately displayed on the computer screen, where the measurement procedure would be repeated if the quality of the recording is of poor quality (due to patient incoordination). The mandible would start moving from the reference position, reaching maximum amplitude before returning to the reference position. In total, two recordings were saved and stored for each type of movement. The device allows for the superposition of curves, which enables the verification of the reproducibility of joint movements (9).

Diagnosis was made in accordance with the graphic records, which were interpreted as follows: i) Normal TMJ was defined as clear, regular, superimposed curves, with $\geq 14$ mm quantity for opening, $9 \mathrm{~mm}$ for maximum propulsion and laterotrusion (Fig. 3); ii) TMJ with DDwR was defined as observations that the advancement and return pathways to the reference position do not overlap, but movement is not limited from a quantitative point of view (Fig. 4); and iii) TMJ with DDwoR was defined as significant quantitative limitation, with straight, linear pathways (Fig. 5). In addition, Bennett angle values and the condylar sagittal inclination were also measured using computerized axiography.

MRI examination. All MRI examinations were performed using a 1.5-T MRI equipment (Siemens Avanto; Siemens Healthineers). MRI assessment was performed by a radiologist (NB) blinded to both the clinical and axiographic findings. Bilateral MR imaging of the TMJs was performed using the same protocol. The data were collected on a 205/256 matrix, with a field of view of $120 \mathrm{~mm}$ and a flip angle of 150 degrees. Oblique-sagittal and coronal images were obtained of both closed- and opened-mouth positions, perpendicular and parallel to the long axis of the condyle. The MRI protocol included T2-weighted and proton density sequences, with the thickness of slices at $3 \mathrm{~mm}$.

Disc displacement was considered to be present if the posterior margin of the posterior band was situated anteriorly to the vertical orientation of the condyle (the ' 12 o'clock line') (10). The MRI examination was performed within 1-7 days following clinical examination and computerized axiography. The results were interpreted by a single specialist in maxillofacial imaging (NB; Figs. 6 and 7).

Statistical analysis. The characteristics of diagnostic tests (computerized axiography being the index test, whilst MRI 


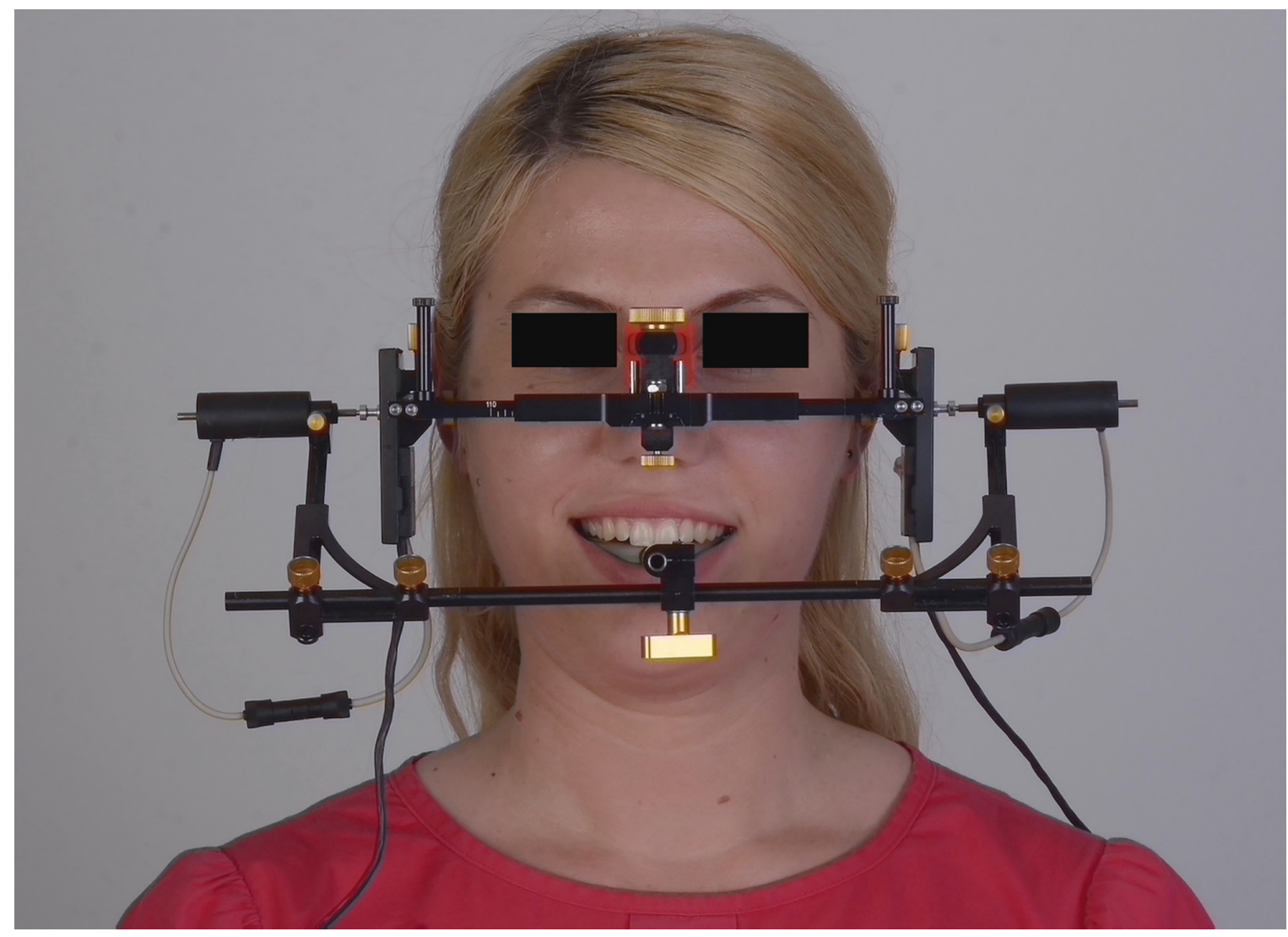

Figure 1. Frontal view of the computerized axiograph mounted onto the face of the patient.

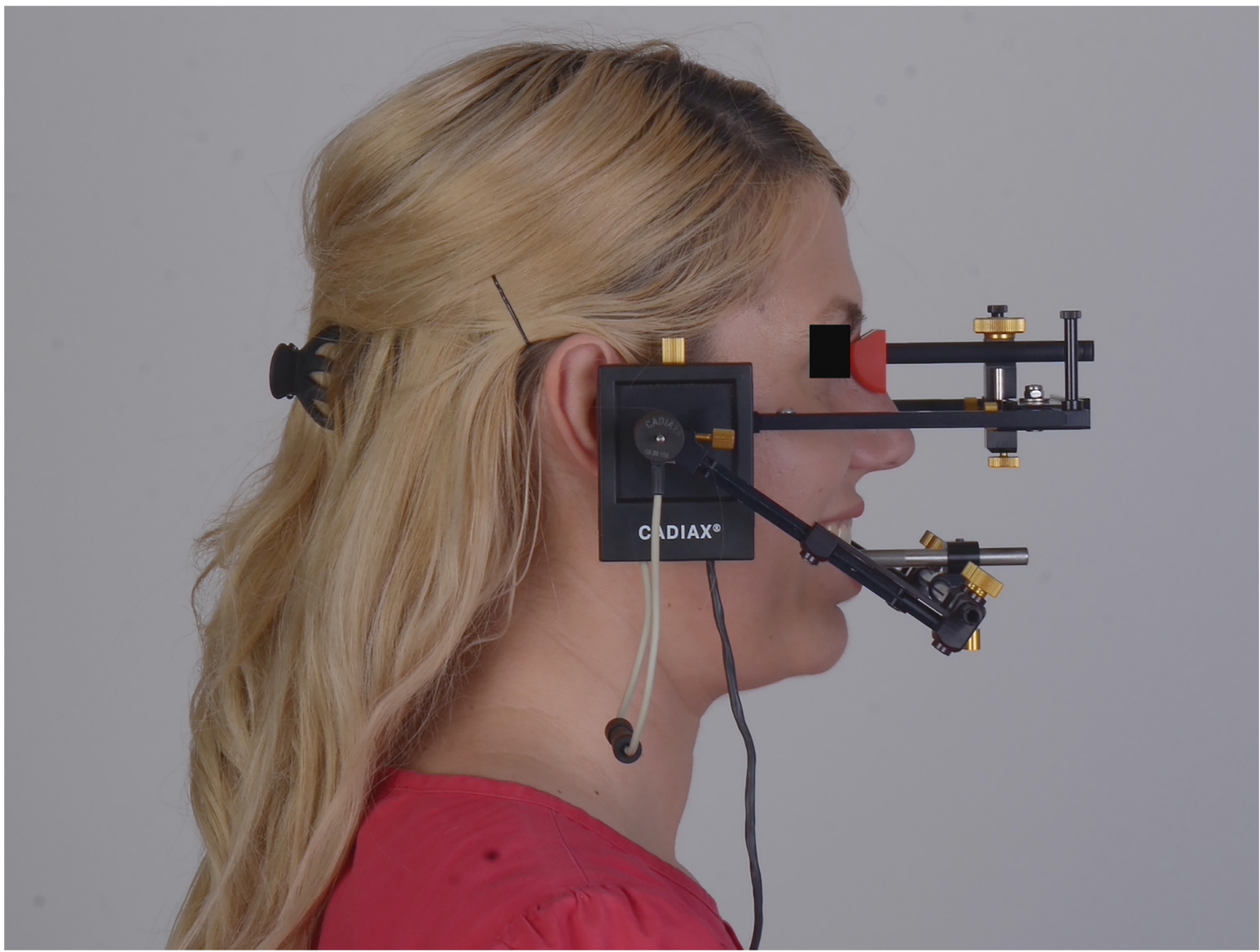

Figure 2. Lateral view of the computerized axiograph mounted onto the face of the patient. 


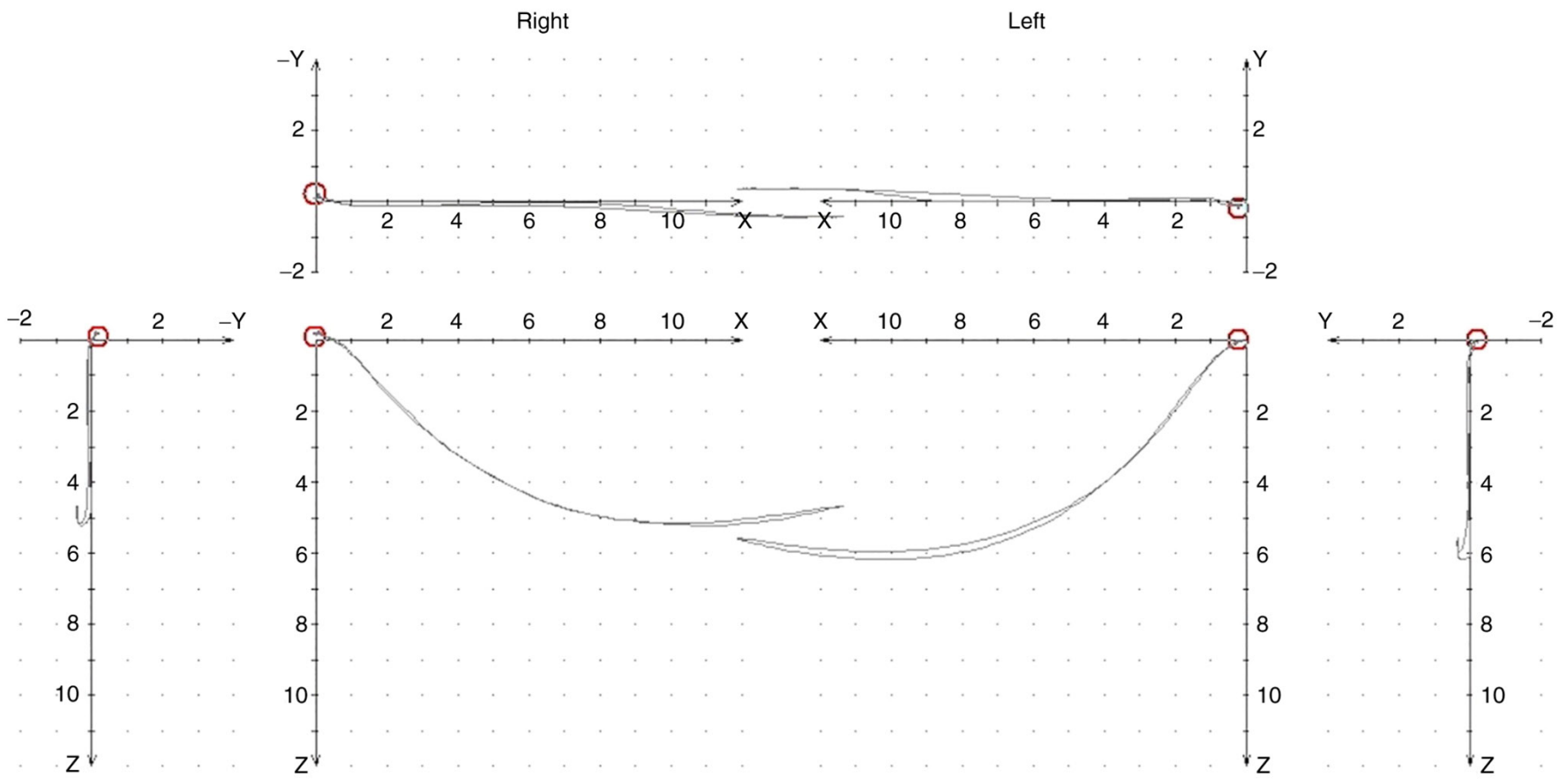

Figure 3. Axiographic pathway for the opening-closing movements of a healthy temporomandibular joint. Clear, overlapping, quantitatively normal ( $\geq 14$ mm quantity for opening) pathways can be observed. The x-axis- sagittal and horizontal. The y-axis- transverse and horizontal. The z-axis- frontal and vertical. The red circles represent the starting point.

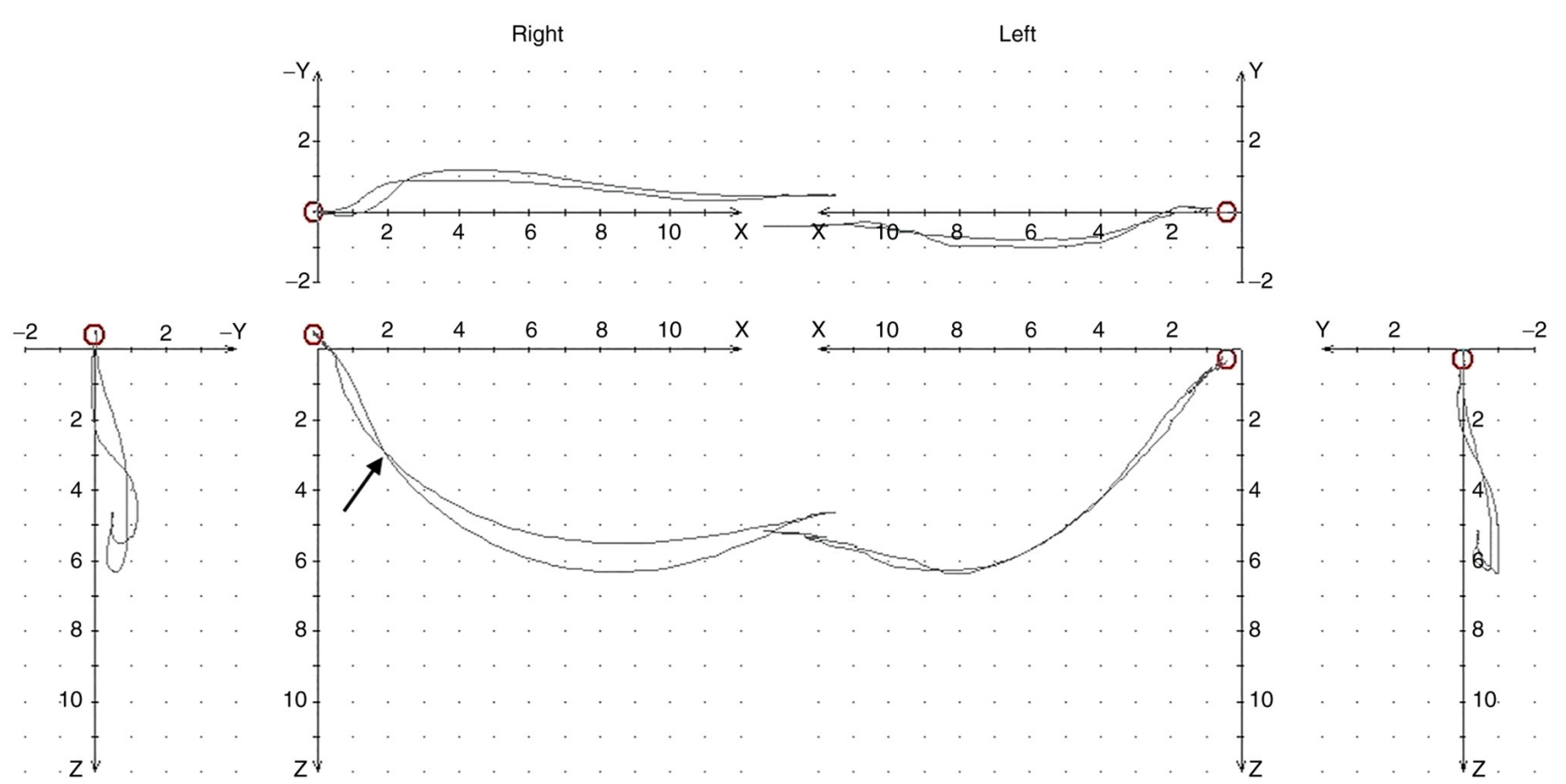

Figure 4. Axiographic pathway for the opening-closing movements of a temporomandibular joint with disc displacement with reduction. The lack of superposition of the opening and closing pathways on the right side can be observed. The x-axis- sagittal and horizontal. The y-axis- transverse and horizontal. The z-axis- frontal and vertical. The red circles represent the starting point. Clicking appears at the point of intersection of the two pathways (arrow).

being the standard test), namely sensitivity, specificity, positive and negative predictive values, accuracy, Youden index and the $95 \%$ confidence intervals (CI), were all computed. Exact binomial confidence limits were calculated for the sensitivity, specificity, positive and negative predictive values. Positive and negative likelihood ratios were also assessed. All analyses were performed using the $\mathrm{R}$ environment for statistical computing and graphics, version 3.2.3 (11).

\section{Results}

Comparison of the three techniques. Clinical examination revealed 23 (34.85\%) TMJs with DDwR, 18 (27.27\%) TMJs 


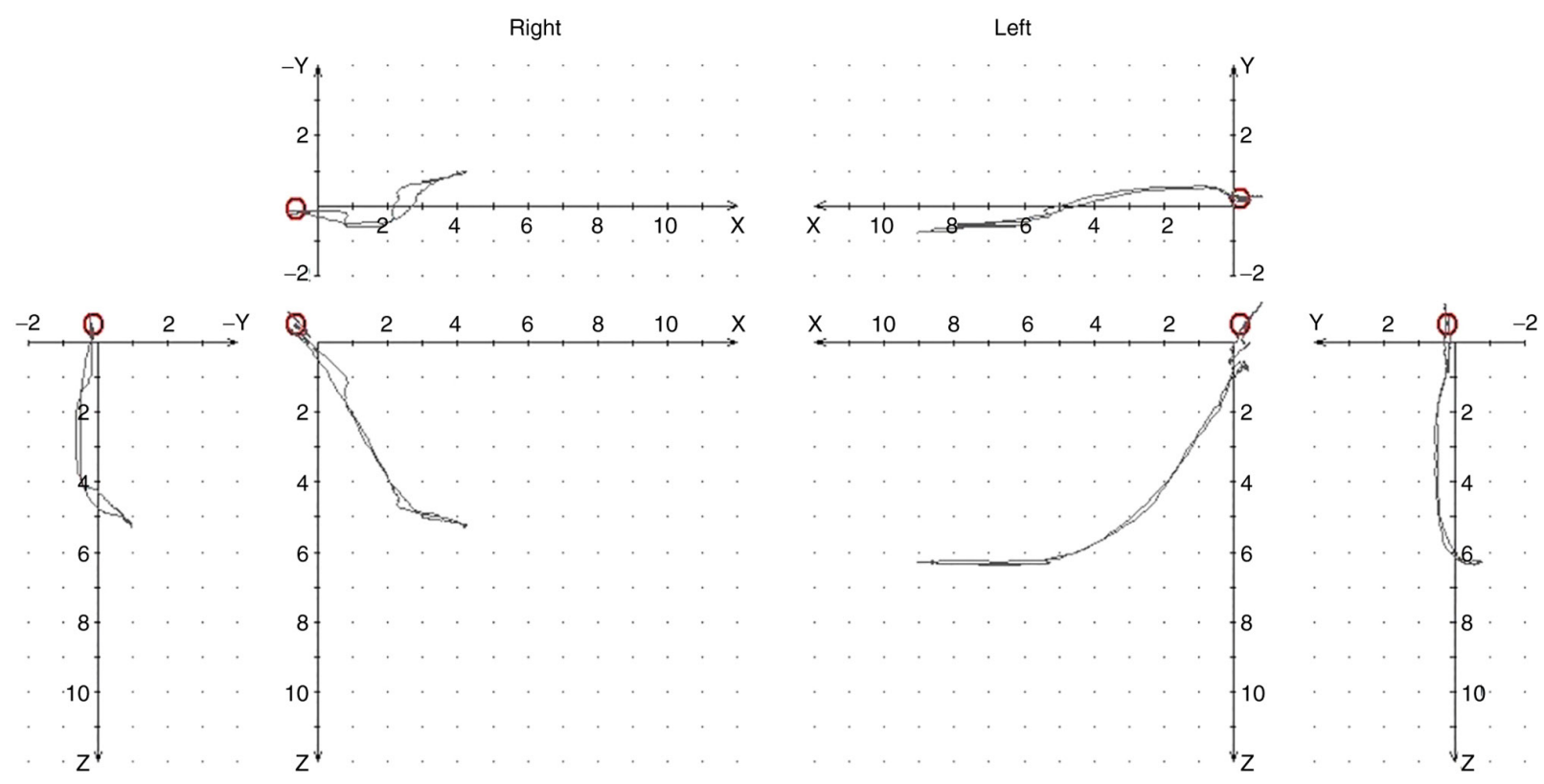

Figure 5. Axiographic pathway for the opening-closing movements of a temporomandibular joint with disc displacement without reduction. The pathway on the right side is quantitatively reduced and completely straight, lacking the specific concavity of a normal pathway. The x-axis- sagittal and horizontal. The $\mathrm{y}$-axis- transverse and horizontal. The z-axis- frontal and vertical. The red circles represent the starting point.
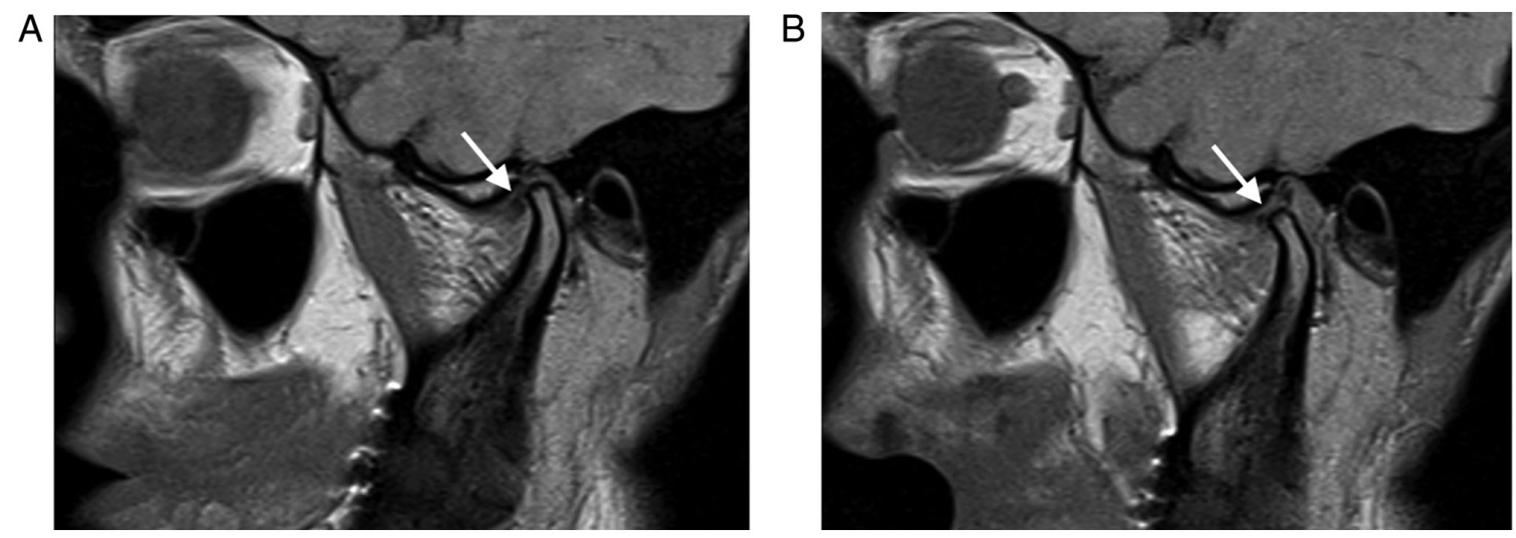

Figure 6. Sagittal proton density MRI image of a normal temporomandibular joint. (A) Mouth-closed. (B) Mouth-opened. The disc (arrow) is in the correct position.
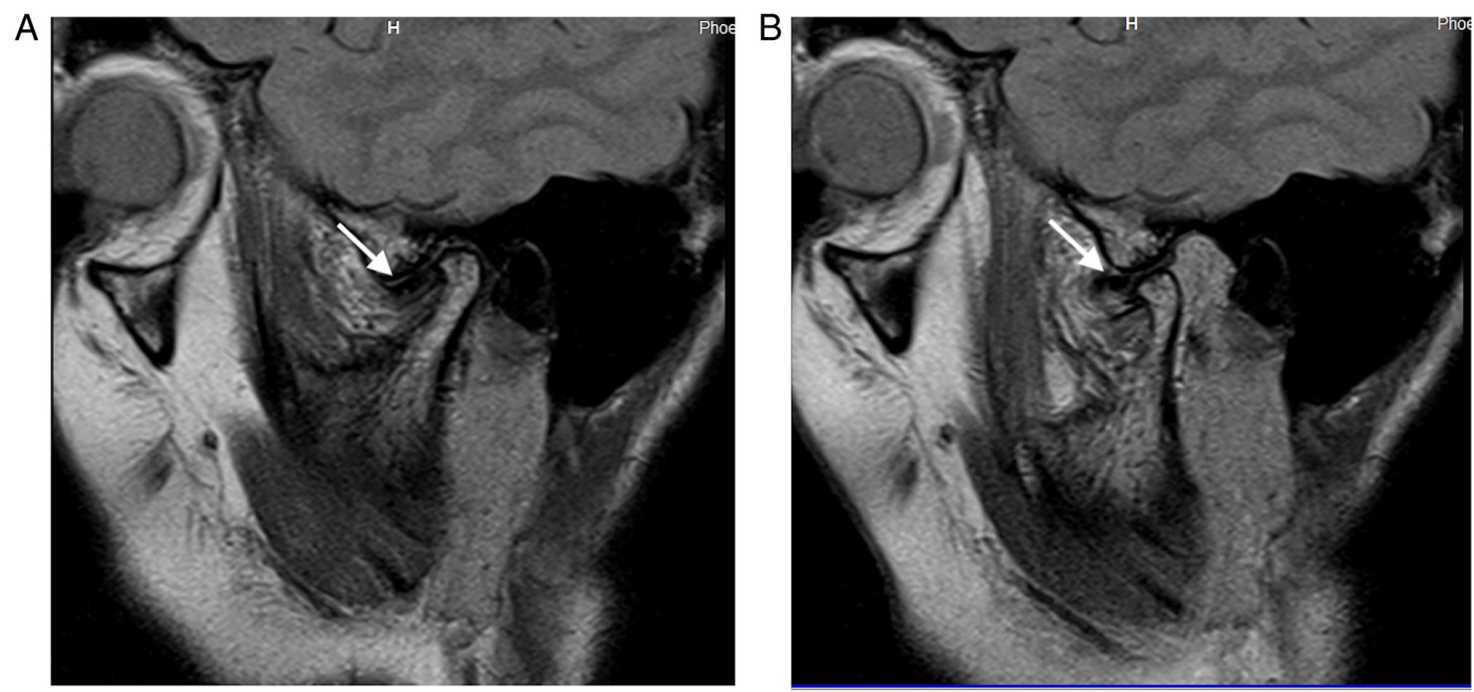

Figure 7. Sagittal proton density MRI image of an anterior disc displacement without reduction. (A) Mouth-closed. (B) Mouth-opened. Note the displaced disc (arrow) when the mouth is closed does not return to its normal position at the maximal mouth opening position 
Table I. The results of the clinical examination, computerized axiography and MRI.

Examination

Total $\%$ of disc displacements

Total $\%$ of normal temporomandibular joints

62.12

65.15

71.21
37.88

34.85

28.79
Table II. Computerized axiography findings compared with MRI findings.

\begin{tabular}{lcc}
\hline Statistical parameter & $\begin{array}{c}\text { Disc } \\
\text { displacement }\end{array}$ & $\begin{array}{c}95 \% \text { confidence } \\
\text { interval }\end{array}$ \\
\hline Sensitivity, \% & 85.11 & $71.69-93.8$ \\
Specificity, \% & 94.74 & $73.97-99.87$ \\
PPV, \% & 97.56 & $87.14-99.94$ \\
NPV, \% & 72 & $50.61-87.93$ \\
LR $^{+}$ & 16.17 & $2.39-109.36$ \\
LR $^{-}$ & 0.16 & $0.08-0.31$ \\
Diagnostic accuracy, \% & 87.88 & $77.51-94.62$ \\
Youden index & 0.8 & $0.46-0.94$ \\
Number necessary for & 1.25 & $1.07-2.19$ \\
diagnosis & & \\
\hline
\end{tabular}

PPV, positive predictive value; NPV, negative predictive value; $\mathrm{LR}^{+}$, positive likelihood ratio; $\mathrm{LR}^{-}$, negative likelihood ratio.

with DDwoR and 25 (37.88\%) healthy TMJs. Computerized axiography detected $22(33.33 \%)$ TMJs with DDwR, $21(31.82 \%)$ TMJs with DDwoR and $23(34.85 \%)$ healthy TMJs. MRI identified 30 (45.45\%) TMJs with DDwR, 17 (25.76\%) TMJs with DDwoR and 19 (28.79\%) healthy TMJs. In total, clinical examination revealed 41/66 (62.12\%) TMJs with disc displacements, whereas computerized axiography showed 43/66 (65.15\%). MRI indicated 47/66 (71.21\%) TMJs with disc displacements (Table I). The number of disc displacements diagnosed clinically and that diagnosed using computerized axiography are comparable (41 vs. $43 \%$; Table I). MRI, being the gold standard for disc visualization, revealed the real number of disc displacements, which was 47.

Statistical indicators of computerized axiography, including the sensitivity, specificity, diagnostic accuracy, Youden index, positive predictive value, negative predictive value, positive likelihood ratio and negative likelihood ratio were assessed, using MRI as a reference standard (Table II). The sensitivity and specificity of computerized axiography for detection of TMJ disc displacements were 85.11 and $94.74 \%$, respectively. The Youden index was also found to be high (0.8), showing a viable diagnostic accuracy for computerized axiography.

The values of the Bennett angle and of the sagittal condylar inclination were also calculated. The sagittal condylar inclination had a mean \pm SD of 42.08 \pm 8.02 (95\% CI, 40.1-44.05), with values ranging from 20 to 60 . The Bennett angle variable (transversal condylar guidance) had a median (IQR) of 9 (7-13) with values ranging from 5 to 30 (95\% CI, 7.5-11).

\section{Discussion}

The patient's clinical history and the observation of articular noises, such as clicking, are typically used to diagnose DDwR clinically (12). However, clicking may be the result of other causes, not only disc displacement (13). For instance, articular ligaments may produce such noises, where a clicking sound may occur during the rotational movement of the collateral lateral ligament due to its fragility (13). Synovial noises, which occurs especially when chewing solid food, are also described as having the characteristics of clicking sounds. In addition, condylar surface irregularities may be the cause of articular noises during the rotational movement occurring in the lower compartment of the joint, between the condylar surface and the articular disc $(12,14)$. Such cases are difficult to diagnose clinically, rendering the axiographic investigation of the TMJ potentially beneficial for obtaining additional important data for accurate clinical diagnosis.

The high statistical values of specificity and sensitivity obtained in the present study suggest computerized axiography to be an important screening method for confirming or exclude disc displacement. The resulting Youden index also exhibited diagnostic value for the use of computerized axiography. However, incipient DDwR cannot be diagnosed using axiography due to the thickness of the bite fork applied on the lower arch. The presence of the fork slightly increases the vertical dimension of occlusion, where if the displacement is mild, the disc would adjust itself to the new vertical dimension so that it appears as 'normal' in the reference position according to the axiograph recording. The present study reported six such false negative cases. In this situation, accurate diagnosis was required using MRI examination.

Previous studies performed by Ahangari et al (15) and Torabi et al (16) substantiated the accuracy of the Cadiax Compact $^{\circledR}$ II device recordings, in terms of both the resulting graphical output and values used for programming the semi-adjustable articulator. In particular, the semi-adjustable articulator program were compared to the wax records used for programming the articulator.

Controversies remain regarding the application of electronic devices for diagnosing TMJ disorders. Electronic methods include axiography, electromyography and Doppler ultrasound for recording articular noises (17). A number of previous accounts (18-20) asserted that using devices for recording mandibular dynamics serve no diagnostic value. Cooper and Rabuzzi (21) reported that any movements recorded by axiography are slower in patients with TMD compared with those in healthy individuals $(<300 \mathrm{~mm} / \mathrm{sec})$. By contrast, Feine et al (22) found that slower speeds, even bellow the aforementioned value, can also be recorded in healthy individuals. In addition, 
Table III. Results of the main studies that previously evaluated the sensitivity and specificity of axiography compared with MRI results.

\begin{tabular}{lcccc}
\hline Author & Year & Sensitivity, $\%$ & Specificity, \% & (Refs.) \\
\hline Parlet et al & 1993 & 64 & 100 & $(26)$ \\
Rammelsberg et al & 1996 & 86 & 90 & $(27)$ \\
Ozawa and Tanne & 1997 & 40 & 94 & $(28)$ \\
Bracco et al & 1997 & 56 & 96 & $(29)$ \\
Kobs et al & 2004 & 80 & 90 & $(30)$ \\
\hline
\end{tabular}

Romanelli et al (23) proposed that axiography is an accurate method for diagnosing disc displacements in the TMJ. Due to insufficient clinical data being available in this field, in a 2018 review Constantinides et al (24) concluded that computerized axiography is not a useful tool for detecting internal TMJ disturbances. In a previous study of Manfredini et al (25), which evaluated the diagnostic value of axiography in disc displacements and joint effusion, reached the same conclusion. Indeed, controversial opinions and findings have been reported on the potential diagnostic value of axiography. The values obtained for sensitivity and specificity are summarized in Table III. All previous studies (26-30) presented in Table III indicate a very good specificity for computerized axiography, which is in agreement with the results from the present study. Axiography appeared to be a highly specific form of TMJ examination. In terms of sensitivity, it appears to be lower (between 56 and 86\%). As also found in the present study and mentioned by Ozawa and Tanne (28), the false negative results are likely to be due to the application of the bite fork, which could change the condylar starting position and is an important factor in cases with incipient TMJ disc displacements. In the present study, only six such cases were reported and the sensitivity for the overall diagnosis of disc displacements was $85.11 \%$. In addition, the diagnostic sensitivity obtained in the studies mentioned in Table III could be dependent on the multiple systems of classification used for TMJ disc displacements (in terms of clinical and MRI classification). The classification system differs from one study to another and could affect the values of statistical indicators.

Piancino et al (31) previously analyzed the association between computerized axiography and MRI data, which yielded a very low kappa index of $13 \%$. They then concluded that MRI and computerized axiography are two completely different types of examination methods that should instead be used in conjunction with one another (31). The same conclusion has also been reached by Piehslinger et al (32), who obtained a concordance of only $45 \%$ between MRI and computerized axiography.

In the present study, a mean value of the sagittal condylar inclination of 42 degrees was found, whereas the Bennett angle was 9 degrees. However, these values obtained were heterogeneous, rendering correlation analysis between these two parameters and the TMJ pathological parameters impossible. However, a significant difference between a normal TMJ on one side and a displaced TMJ on the other side for the same subject, regarding the Bennett angle and the sagittal condylar inclination, was found.
Computerized axiography can also be used as a tool for monitoring treatment progression by analyzing the graphic outputs at different times, as suggested by a previous study performed by Piancino et al (33). However, similar types of data can be obtained by clinical examination of the muscles and TMJ (17).

A number of studies $(30,34)$ emphasized the importance of associating clinical examination parameters with computerized axiography, suggesting that axiography should be used as an extension of clinical examination as part of the clinical analysis. As a result, axiography can increase the diagnostic value to contribute to the differential diagnosis of intra-articular pathology $(32,34)$. The present study proposes that computerized axiography, when used as an additional diagnostic strategy, should be incorporated into the clinical examination procedure. However, a limitation of the present study is that the sample size is small and that the calculation of intra- and inter-examiner agreement was not performed.

To conclude, computerized axiography is a simple and fast method that is capable of reproducing the functional movement of the TMJ, which offers the opportunity to perform comparative analyzes of the two joints. In addition, it can also be used for the periodic evaluation of treatment progression of TMJ pathology. MRI and computerized axiography can produce different types of data regarding the TMJ status. Whilst MRI can yield important structural data, axiography can reveal data on TMJ function.

\section{Acknowledgements}

Not applicable.

\section{Funding}

No funding was received.

\section{Availability of data and materials}

The datasets used and/or analyzed during the current study are available from the corresponding author on reasonable request.

\section{Author's contributions}

DT and SB confirm the authenticity of all the raw data. DT: clinical investigation, conceptualization and analysis of the results. NB: MRI interpretation and MRI image acquisition. DL: methodology design of the study and statistical analysis. IAT: 
visualization and validation. SB: interpretation of data, study design. All authors read and approved the final manuscript.

\section{Ethics approval and consent to participate}

The research protocol was analyzed and approved by the Ethics Committee of the University of Medicine and Pharmacy 'Iuliu Hatieganu’ Cluj-Napoca (approval no. 403/02.07.2015).

\section{Patient consent for publication}

Written informed consent was obtained from each participant enrolled in the study.

\section{Competing interests}

The authors declare that they have no competing interests.

\section{References}

1. Botos AM, Mesaros AS and Zimbran AI: The contribution of computerized axiography to the functional evaluation of the temporomandibular joint: A case report. Clujul Med 89: 438-442, 2016.

2. Slavicek R: Clinical and instrumental functional analysis for diagnosis and treatment planning. Part 5. Axiography. J Clin Orthod 22: 656-667, 1988.

3. Kraljević S, Pandurić J, Badel T and Dulcić N: Registration and measurement of opening and closing jaw movements and rotational mandibular capacity by using the method of electronic axiography. Coll Antropol 27 (Suppl 2): S51-S59, 2003.

4. Mantout B, Giradeau A, Perez C, Re JP and Orthlieb JD Technical validation of a computerized condylographic system. J Stomat Occ Med 1: 45-60, 2008.

5. Buduru S, Blahuc S, Ciumasu A, Kui A, Ciobanu C, Almasan O, Manziuc $M$ and Negucioiu M: Temporomandibular dysfunction diagnosis by means of computerized axiography. Med Pharm Rep 93: 416-421, 2020.

6. Celar A and Tamaki K: Accuracy of recording horizontal condylar inclination and bennett angle with the cadiax compact. J Oral Rehabil 29: 1076-1081, 2002.

7. Schierz O, Klinger N, Schon G and Reissmann DR: The reliability of computerized condylar path angle assessment. Int J Comput Dent 17: 35-51, 2014 (In English, German).

8. Schiffman E, Ohrbach R, Truelove E, Look J, Anderson G, Goulet JP, List T, Svensson P, Gonzalez Y, Lobbezoo F, et al International RDC/TMD consortium network, international association for dental research; orofacial pain special interest group, international association for the study of pain. Diagnostic criteria for temporomandibular disorders (DC/TMD) for clinical and research applications: Recommendations of the international RDC/TMD consortium network and orofacial pain special interest group. J Oral Facial Pain Headache 28: 6-27, 2014.

9. Madhavan S, Dhanraj M and Jain AR: Methods of recording mandibular movements-A review. Drug Invention Today 10: 1254-1259, 2018.

10. Tasaki MM and Westesson PL: Temporomandibular joint: Diagnostic accuracy with sagittal and coronal MR imaging. Radiology 186: 723-729, 1993.

11. R Core Team R: A Language and Environment for Statistical Computing R Foundation for Statistical Computing: 2020. http:// www.r-project.org/index.html (accessed on 21st of October 2017)

12. Okeson JP: Joint intracapsular disorders: Diagnostic and nonsurgical management considerations. Dent Clin North Am 51: 85-103, 2007.

13. Slavicek R: Diagnostics (Chapter 4). In: The Masticatory Organ: Functions and Dysfunctions. Gamma Medizinisch-Wissenschaftliche Fortbildungs-Gmbh, Klosterneuburg, pp398-409, 2006.
14. Bag AK, Gaddikeri S, Singhal A, Hardin S, Tran BD, Medina JA and Curé JK: Imaging of the temporomandibular joint: An update. World J Radiol 6: 567-582, 2014.

15. Ahangari AH, Torabi K, Pour SR and Ghodsi S: Evaluation of the Cadiax Compact ${ }^{\circledR}$ II accuracy in recording preadjusted condylar inclinations on fully adjustable articulator. J Contemp Dent Pract 13: 504-508, 2012.

16. Torabi K, Pour SR, Ahangari AH and Ghodsi S: A clinical comparative study of cadiax compact II and intraoral records using wax and addition silicone. Int J Prosthodont 27: 541-543, 2014.

17. Manfredini D: Etiology. Instrumental approach, Part II. In: Current Concepts on Temporomandibular Disorders. Quintessence Publishing Co. Ltd., London, pp223-235, 2010.

18. Widmer CG, Lund JP and Feine JS: Evaluation of diagnostic tests for TMD. J Calif Dent Assoc 18: 53-60, 1990.

19. Theusner J, Plesh O, Curtis DA and Hutton JE: Axiographic tracings of temporomandibular joint movements. J Prosthet Dent 69: 209-215, 1993

20. Greene CS: Can technology enhance TM disorder diagnosis? J Calif Dent Assoc 18: 25-33, 1990.

21. Cooper BC and Rabuzzi DD: Myofacial pain dysfunction syndrome: A clinical study of asymptomatic patients. Laryngoscope 94: 68-75, 1984.

22. Feine JS, Hutchins MO and Lund JP: An evaluation of the criteria used to diagnose mandibular dysfunction with the mandibular kinesiograph. J Prosthet Dent 60: 374-380, 1988.

23. Romanelli GG, Harper R, Mock D, Pharoah MJ and Tenenbaum HC: Evaluation of the temporomandibular joint internal derangement. J Orofac Pain 7: 254-262, 1993.

24. Costantinides F, Parisi S, Tonni I, Bodin C, Vettori E, Perinetti G and Lenarda RD: Reliability of kinesiography vs magnetic resonance in internal derangement of TMJ diagnosis: A systematic review of the literature. Cranio 38: 58-65, 2018.

25. Manfredini D, Favero L, Federzoni E, Cocilovo F and Guarda-Nardini L: Kinesiographic recordings of jaw movements are not accurate to detect magnetic resonance-diagnosed temporomandibular joint (TMJ) effusion and disk displacement: Findings from a validation study. Oral Surg Oral Med Oral Pathol Oral Radiol 114: 457-463, 2012.

26. Parlett K, Paesani D, Tallents RH and Hatala MA: Temporomandibular joint axiography and MRI findings: A comparative study. J Prosthet Dent 70: 521-531, 1993.

27. Rammelsberg P, Pospiech P, May HC and Gernet W: Evaluation of diagnostic criteria from computerized axiograhy to detect internal derangements of the TMJ. Cranio 14: 286-295, 1996.

28. Ozawa S and Tanne K: Diagnostic accuracy of sagittal condylar movement patterns for identifying internal derangement of the temporomandibular joint. J Orofac Pain 11: 222-231, 1997.

29. Bracco P, Dergibus A, Piscetta R and Giaretta GA: TMJ clicking: A comparison of clinical examination, sonography and axiography. Cranio 15: 121-126, 1997.

30. Kobs G, Bernhardt O and Meyer G: Accuracy of computerized axiography controlled by MRI in detecting internal derangements of the TMJ. Stom Baltic Dent Maxillofac J 6: 7-10, 2004.

31. Piancino MG, Cirillo S, Frongia G, Cena F, Bracco AA and Bracco P: Axiography and MRI in the diagnosis of temporomandibular joint pathology. J Stomat Occ Med 2: 50-51, 2009.

32. Piehslinger E, Schimmerl S, Celar A, Crowley C and Imhof H: Comparison of magnetic resonance tomography with computerized axiography in diagnosis of temporomandibular joint disorders. Int J Oral Maxillofac Surg 24: 13-19, 1995.

33. Piancino MG, Loberi L, Frongia G, Reverdito M, Slavicek R and Bracco P: Computerized axiography in TMD patients before and after therapy with 'function generating bite'. J Oral Rehabil 35: 88-94, 2008.

34. Giraudeau A, Cheynet F, Mantout B, Dejou J, Sarrat P and Orthlieb J: Diagnostic of intracapsular derangement of TMJ: comparison of clinical examination and condylography with MRI. Stomatologie 104: 154-167, 2007.

This work is licensed under a Creative Commons Attribution-NonCommercial-NoDerivatives 4.0 International (CC BY-NC-ND 4.0) License. 